\title{
Policies for a resource efficient economy in Europe: findings from the POLFREE project
}

\author{
Paul Ekins*a $^{* a}$ Teresa Domenech ${ }^{\mathrm{b}}$, Paul Drummond \\ University College London, Central House, 14 Upper Woburn Place, London WC1H 0NN, United Kingdom \\ *Corresponding author; a: p.ekins@ucl.ac.uk; b: t.domenech@ucl.ac.uk; c: p.drummond@ucl.ac.uk
}

This Special Issue of Ecological Economics contains eight papers derived from the research carried out under the European $7^{\text {th }}$ Framework Programme for Research (FP7) project, Policy Options for a Resource-Efficient Economy (POLFREE), which ran from October 2012 to April 2016". According to the project summary: "The project will construct a theoretical framework for the analysis of resource efficiency, with detailed comparison of the trends and policies at EU and Member State (MS) level, and an analysis of business barriers to resource efficiency; thereby developing an enhanced understanding of the drivers of inefficient resource use. This will lead to an exploration of new concepts and paradigms that can bring about a radical increase in resource efficiency, and a vision for a resource-efficient economy in the EU, with suggestions also for new more resource-efficient business models for firms. In addition, ideas for a global governance regime that can promote resource-efficient economies among the EU's trading partners and more widely will be explored. From its new vision for a resource-efficient Europe, the project will propose new policy mixes, business models and mechanisms of global governance through which resource-efficient economies may be promoted. This will lead in turn to work on creating, modelling and visualising scenarios for the emergence of resource-efficient economies, through linking quantitative economic and ecological models, and simulating the policies and policy mixes derived in the earlier work. The scenarios and associated policy analysis will be given an integrated interpretation across economic, ecological and social dimensions." The papers in this Special Issue contain the main results of the project. Further information about POLFREE may be found on the POLFREE website (https://www.ucl.ac.uk/polfree), while the project's full findings are contained in the publications at https://www.ucl.ac.uk/polfree/publications.

The background to the POLFREE project was the publication in 2011 by the European Commission of a 'Roadmap to a Resource-Efficient Europe' by 2050, a key component of the 'Resource-Efficient Europe Flagship Initiative', which was itself part of the Europe 2020 strategy. The overarching objective of this Roadmap was to lay the foundations to achieve the following vision: "By 2050 the EU's economy has grown in a way that respects resource constraints and planetary boundaries, thus contributing to global economic transformation. Our economy is competitive, inclusive and provides a high standard of living with much

${ }^{1}$ Grant agreement No.308371 
lower environmental impacts. All resources are sustainably managed, from raw materials to energy, water, air, land and soil. Climate change milestones have been reached, while biodiversity and the ecosystem services it underpins have been protected, valued and substantially restored."

The data show that there is still a very long way to go if this vision is to be realised. Although over 2000-2009 a number of countries showed absolute decoupling between their Gross Domestic Product (GDP) and Domestic Material Consumption (DMC), the trend is much less pronounced when the metric Raw Material Input (RMI) is used, with RMI taking account of the extent to which material provisioning in the EU has not been reduced, but outsourced to other countries (Kemp et al. 2014, Figures 1 and 2, pp.13-14).

POLFREE research soon made it clear that there were many barriers to achieving the ambitious vision of the EU Roadmap. These barriers interacted and reinforced each other as a 'web of constraints' (Kemp and Dijk, 2013; Kemp et al., 2014)), "that include individual and institutional behavioural patterns, inertia and direct and indirect interconnections between the institutional, social and individual levels. An implication of this is that the design of a farreaching policy strategy on resource efficiency requires systemic changes operating at different levels including business models, social consumption patterns, regulation and discourses." (Kemp et al. 2014, p.7).

In pursuit of the vision of increasing resource efficiency, both the EU and its Member States (MSs) have introduced over a number of years a wide range of relevant policies. Domenech and Bahn-Walkowiak (2018, this issue) review and assess the scope and effectiveness of these policies. At the EU level, these policies are motivated by three important strategy documents: "the Europe 2020 strategy for smart, sustainable and inclusive growth; the flagship initiative on resource efficiency and the resource efficiency roadmap; and the circular economy package" (ibid., p.4). However, policy frameworks for resource efficiency (RE) and the circular economy (CE) are complex, often fragmented, and affected by competing visions and goals across policy domains and levels of governance, including EU and MS, as illustrated in Figure 1. 


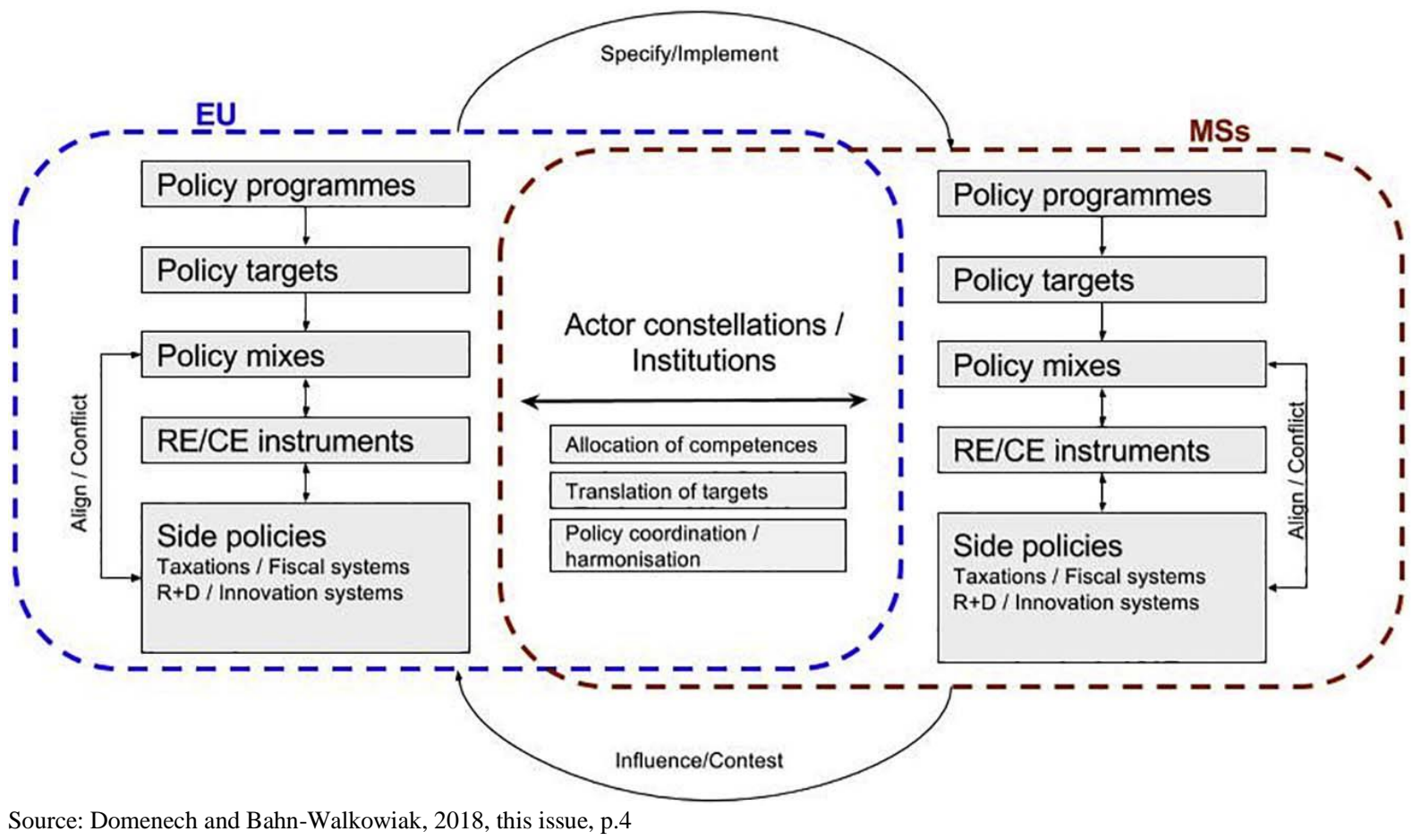

The proposal for an EU resource efficiency/productivity target was dropped from the EU circular economy package, but nine EU MSs have such targets, and three (Austria, Germany and Finland) "have a dedicated strategy for resource efficiency" (ibid., p.8). The policies of four MSs (Austria, German, Hungary and the Netherlands) are explored in some detail to show how MSs have sought to give practical expression to their resource efficiency ambitions. The case countries provide numerous examples of both synergies and conflicts between policies, the most egregious of conflicts being the continuing subsidisation of environmentally damaging and resource-inefficient activities.

Domenech and Bahn-Walkowiak's conclusion of their investigation is that: "The analysis of the EU policy framework and national policies on resource efficiency has revealed a complex policy picture made up of policy strategies, targets and instruments that do not always align across different dimensions of resource efficiency or sectors of activity." (ibid, p.12)

As noted above, the 'web of constraints' that hinders substantial increases in resource efficiency is generated within and between the institutional, social and individual levels. At the individual level, Kammerlander (2014) investigated behavioural barriers to resource efficiency, using three theoretical frameworks (social practices approaches, the concept of society-nature relationships and Bamberg's stage model of self-regulated behavioural change), in respect of three major resource-using consumption domains of personal life food, mobility and housing - and through qualitative interviews, focus groups and a household survey in Austria and Hungary and, for housing, in the Netherlands as well. The interviews indicated that major barriers to increased resource efficiency arose from both socioeconomic factors (e.g. costs, income, availability and time) and personal characteristics (such as lack of awareness and knowledge, laziness, and a desire for comfort and convenience). The focus groups clearly identified interactions between these and some other factors, such as social acceptance, norms and social status, habits, practices and traditions, and lack of infrastructure facilitating resource efficiency, as contributing to the 'web of 
constraints' that hindered moves towards greater resource efficiency. The survey showed that socio-demographic factors (gender, age, educational background, occupation and income) "play the largest role in people's practices and viewpoints" (ibid., p.117) but that there was no consistency across these factors in different domains, or across the countries included in the survey, in respect of (reported) resource-efficient behaviours. The survey further showed that while there was general support for resource efficiency policies involving education and information, there was "little confidence in and desire for structural changes, such as tax and incentive schemes" (ibid., p.118) Such responses suggest that implementing policies that lead to the step-changes in resource efficiency envisaged in the EU Roadmap will be far from easy to achieve and politically controversial.

If Kammerlander (2014) identified the principal barriers to households and individuals taking action to increase their resource efficiency, Diaz et al. (2018, this issue) found similar evidence in respect of business models. They compiled an evidence base of 143 cases of companies which had implemented resource efficiency measures (REMs), from a wide variety of sectors and different countries, categorised according to those which were lifecycle focused (3 kinds of REM), supply side focused (five kinds of REM) and demand side focused (five kinds of REM). These REMs are then related to the business models, and business model change, of the companies that had implemented them, categorised according to their focus on the supply chain, value proposition, customer interface or financial model, as well as to the implementation barriers - institutional, market, organisational, behavioural and technological - which have hindered the introduction of REMs. Finally, these measures and changes are related to the scope (from the single company to the whole value chain) and degree (incremental or radical) of change which they seek to introduce.

Four sets of relationships were mapped: between REMs and business model changes; between REMs and implementation barriers; between REMs and the scope and degree of change and implementation barriers; and between business model changes and implementation barriers. The results are complex. It turns out that companies seek to implement all types of REMs, with no particular bias towards the simpler ones to implement, as might have been expected; $30 \%$ of supply side REMs involve a change in value proposition, while demand side REMs tend to be associated with a change in supply chains and internal processes. With regard to implementation, technical barriers are most important on the supply side, while market barriers are most important for demand side and life cycle REMs. However, all barriers play a role. This suggests an important role for policy to help shape framework conditions conducive to REMs.

The split between radical and incremental resource efficiency innovations is further explored by Tukker and Ekins (2018, this issue), this time in respect of concepts that seek to foster resource efficiency. Some of these concepts are shown in Figure 2, located between the two axes of scope of change and degree of change. 


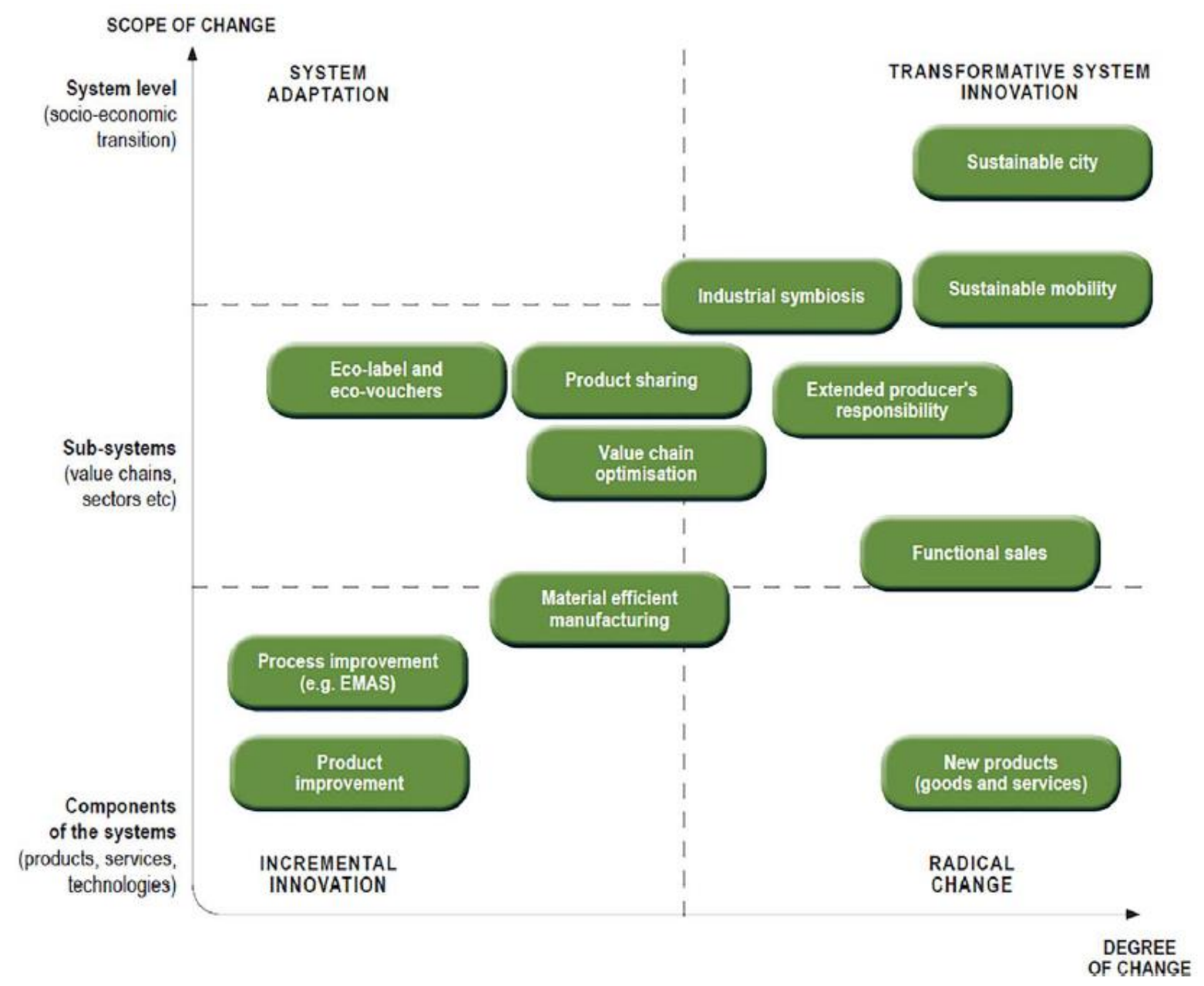

Source: Tukker and Ekins 2018, this issue, Figure 1

The paper then lists the result of a literature analysis of widely used sustainability concepts, which scores them across two dimensions: paradigmatic degree of change (i.e. level of ambition) and plausibility of pathways of change (i.e. feasibility or viability of the proposed change). Those concepts which seem most plausible (which in this analysis include waste prevention, extended producer responsibility, weak and strong sustainability, green growth and green economy, eco-innovation and eco-efficiency, supply chain management, cleaner production, and pollution prevention pays) have a low paradigmatic degree of change; while those concepts embodying a paradigm shift (which in this analysis include ecological economics, Natural Step, small is beautiful, product-service-systems, de-growth, resilience/safe operating space, slow food, transition towns, leasing society, and the Hannover Principles) seem relatively unlikely to be pursued at scale.

It would seem, therefore, that in order for the kinds of step changes in resource efficiency envisaged in the EU Roadmap to come about: either more radical paths will become more implementable; or the aggregation of more incremental changes will in fact open up possibilities for system change; or continuing inefficient resource use in a context of both population and economic growth will lead to real scarcities that force change; or current approaches to resource use will turn out to be more sustainable than envisaged, and are able to continue. In respect of fossil fuel use and climate change, this last option, essentially business-as-usual, has become increasingly unacceptable as the risks of climate change 
become more widely appreciated, and decarbonisation strategies tend to favour the first two options. It remains to be seen whether a similar approach will be taken to resource use more widely.

What is clear is that achieving either incremental or radical change, or both, beyond current patterns of resource use and increases in resource efficiency will require policy interventions at different levels of governance. The global level is relevant because the value chains of many resources, from extraction or cultivation through conversion, production, distribution, use and disposal, now cross both countries and continents. Milligan and O'Keefe (2018, this issue) map the architecture of global resources governance, involving: different actors, including national and local governments, intergovernmental organisations, and for-profit, non-profit and civil society organisations; different normative frameworks; varied behavioural relationships; and different spatial boundaries.

International law provides the basic framework of resource governance through the delineation of the rights and obligations of nation states, which have nominal sovereignty over resources on their territories and in the seas around them. Other arrangements govern resources in the oceans outside coastal waters. In reality, many resources are shared (e.g. ecosystems) or move across jurisdictional boundaries (e.g. water, fish), such that collaborative governance is required, while transnational corporations move resources across such boundaries at scale.

Milligan and O'Keefe (2018, this issue) identify many constraints on efficient resource governance, including (lack of) recognition of local community interests, tensions between international investment agreements and national public interests, corruption, capacity challenges, and uncoordinated decision making. However, there have been numerous attempts to remove these constraints, through coordinated policies towards common goals (for example, the Sustainable Development Goals), and across spatial boundaries, sectors and value chains. The key to the success or otherwise of these initiatives is recognition of the multiple benefits of resources, and the incorporation into decision making processes of the analysis and metrics that enable these to be taken into account.

Although the global level is important for resource governance, it is at the national level that the great majority of policies that use or seek to manage resources are formulated and implemented. Wilts and O'Brien (2018, this issue) explore the policy mixes through which national governments have sought to increase resource efficiency. A policy mix, consisting of coherent mixes of instruments, is seen to be necessary in this context "to overcome a multitude of barriers, to promote innovation across multiple innovation stages, and to effectively address challenges from the global to the local level".

In the analysis of policy mixes, Wilts and O'Brien (ibid.) first stress the importance of analysing the constituent policy instruments, according to some generic criteria, which they identify as stringency, predictability, flexibility, differentiation, depth, and the level of support offered. They then identify from the literature nine relatively resource-intensive domains in which there seem to be substantial opportunities for resource efficiency: phasing out environmentally harmful subsidies, internalising external costs, resource-efficient electricity production and distribution, resource-efficient mobility, resource efficiency in the building sector, minimisation of food losses and wastes, resource efficiency of productservice systems, shifting from waste disposal towards a circular economy and resource efficiency through industrial symbiosis (Wilts and O'Brien 2018, this issue, Figure 1). The 
authors choose three key instruments and analyse their implications for each domain in respect of extraction, production, consumption and end-of-life management, as relevant.

Wilts et al. (2015) gives the full analysis for all nine domains but Wilts and O'Brien in this issue concentrate on just three: environmentally harmful subsidies, food losses and waste, and product-service systems. Through this analysis they identify the key challenges of designing policy mixes as avoiding trade-offs, maximising synergies, and promoting coherence between instruments, and ensuring that they are coordinated across different domains. They suggest that further research is required to determine in different national contexts what institutional structures would best contribute to carrying out these complex tasks.

As mentioned at the start of this Editorial a key motivation for this research was the EU Roadmap to a Resource-Efficient Europe, which envisaged a transformation in the EU's use of resources by 2050. A major objective of POLFREE research was to identify different pathways to this more resource-efficient future, the policies through which it could be achieved, and both its characteristics and the economic implications of achieving it.

The first stage of addressing this objective was to generate a vision of a resource-efficient Europe, the main elements are which are shown in Figure 3.

Figure 3: Main elements of the POLFREE vision for a resource efficient Europe

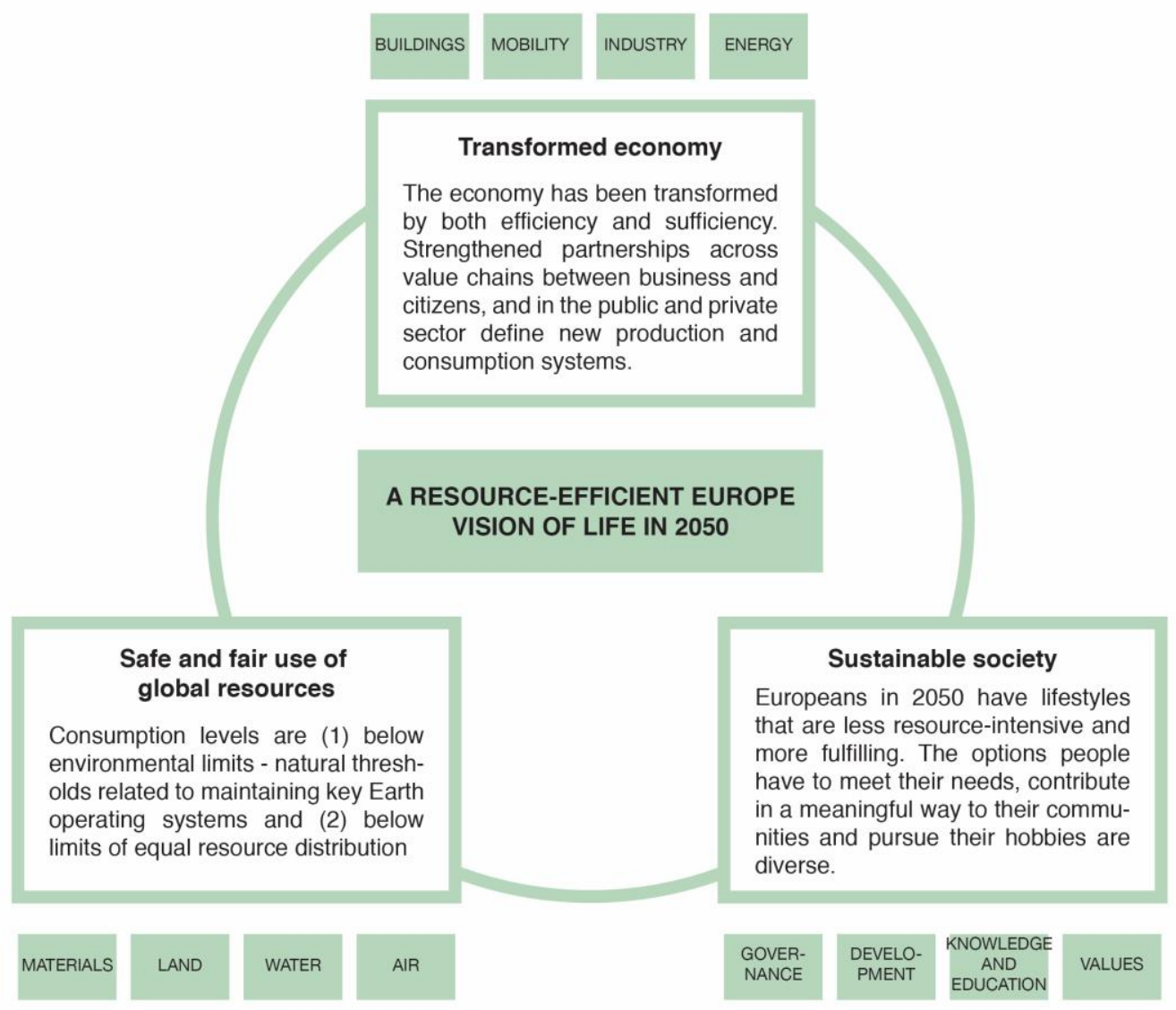


The vision itself was constructed through a literature review, selection of existing sustainability visions for detailed analysis, and several participatory workshops, described in Jäger (2014), from which the quantitative resource targets for the POLFREE were derived, and are shown in Table 1. In addition, a poster visualisation of the vison was produced in the form of a flow diagram, moving from aspirations for increased well-being, through a recognition of planetary boundaries and an acknowledgement of the need for decoupling between economic growth and resource use, to achieve targets for resource use and environmental impacts that ensure that human activities remain with the safe operating space defined by the planetary boundaries. This space implies a resource-efficient economy with certain key characteristics, the achievement of which will depend on putting in place both policies and practices that are very different from those of the economy today. This visualisation may be viewed in Drummond 2015, Annex II. 
2050 Target

5 tonnes Raw Material Consump-
tion (RMC) per capita (global)

No net additions to building stock (EU)

\section{Materials}

Land

Water

Carbon

EU mean global water footprint per capita reduced $30-50 \%$ below 2004 levels

Water Exploitation Index (WEI) below $20 \%$ in all EU countries

EU mean global carbon footprint per capita reduced $60-80 \%$ below 2004 levels

\section{Description}

Returning to a global level of global raw material extraction equivalent to the year 2000 and distributing this level equally among the expected world population in 2050

European demand for primary resources is reduced to the point that they can be nearly all sourced within the built environment through e.g. urban mining. This also implies a reduced land take and much higher levels of renovation of the existing building stock.

Low target: planetary boundary for land use change to limit EU global cropland footprint re- effects of climate change. High duced to $0.17-0.20 \mathrm{ha} / \mathrm{capita}$ target: halt the loss of biodiversity and keep land use change within the safe operating space

No net land take due to expansion of built-up land and no soil degradation (implies long-term maintenance of soil fertility through No net loss of cropland (EU) good agricultural practices to ensure production over the years to come). Overarching rationale is to prevent the loss of fertile cropland in the EU.

The water footprint covers not only the demand consumption of water directly but also the water in imported goods. At $20 \%$ a region is defined as being under "water stress".

Considers the impacts of goods and services imported into the $\mathrm{EU}$

EU GHG emissions reduced by To keep climate change below 80-95\% (below 1990 levels) $2^{\circ} \mathrm{C}$ from pre-industrial levels 
The vision having been established, three different narrative scenarios of how to realise the vision for a resource-efficient and low-carbon Europe were constructed and are described in detail in Schanes et al. (2018, this issue). The three scenarios - Global Cooperation, Europe Goes Ahead, and Civil Society Leads - are based on very difference governance models, worldviews and actors who drive the process, but all of them achieve the targets set out in Table 1 and are consistent with the vision of Figure 5. As the names imply, in Global Cooperation, multilateral processes are the driving forces for increasing resource efficiency, in Europe Goes Ahead, Europe acts as a global forerunner to implement innovation and technological development for resource efficiency, while Civil Society Leads envisages a groundswell of decentralised movements which spurs national governments to adopt resource efficiency policies in the absence of European or global leadership. In each of these contexts, the qualitative scenarios explore the implications for governance, and European industry and innovation, energy, food and agriculture, mobility, and buildings.

These narrative scenarios provide the basis for the quantitative description of the scenarios and their economic and social implications, described in Distelkamp and Meyer (2018, this issue). Formal modelling of resource efficiency issues and their relation to the macroeconomy is still in its relative infancy, with two main classes of models (computable general equilibrium [CGE] and macro-econometric) being employed. The different types of models are based on very different economic theories and assumptions and can therefore give quite different results. Meyer and Ahlert (2018, this issue) discuss these differences in relation to the two models used in the POLFREE project, the macro-econometric GINFORS model, and the CGE EXIOMOD, and provide some explanation as to why the same policy measures in the Global Cooperation scenario cause GINFORS, but not EXIOMOD, to reach the resource efficiency targets in Table 1, and why the results of their modelling the economic implications of these policies diverge quite markedly. In GINFORS GDP, household consumption, public consumption, investment, imports and exports all increase, some quite markedly, whereas in EXIOMOD only household consumption and imports increase, by a lesser percentage, and the other variables are reduced. As an example, in GINFORS GDP in 2050 increases by $7.9 \%$ from the baseline, whereas in EXIOMOD it declines by $0.6 \%$ (Meyer and Ahlert, 2018, this issue, Table 3).

Distelkamp and Meyer (2018, this issue) report simulated policies and results from the GINFORS model for all three of the scenarios. In all scenario exercises of this sort, the characterisation of the baseline (here called the Business-As-Usual [BAU] scenario) is of critical importance, because it is against this that the other scenario results are compared. Under BAU, driven by average global economic growth of $2.1 \%$ and population growth to over 9 billion people by 2050, global $\mathrm{CO}_{2}$ emissions more than double from 1990's level to more than $50 \mathrm{GtCO}_{2}$, and material demands triple from 1990's level to more than $120 \mathrm{Gt}$. The considerable reduction in demands for fossil fuels and other materials in the resource efficiency scenarios induces significant cost reductions, such that in Global Cooperation global GDP in 2050 is 5.2\% above the BAU level, and in Europe Goes Ahead is 8.6\% higher, as reduced material intensity and increased competitiveness stimulate economic growth, particularly European growth and exports in the latter scenario. In Civil Society Leads, a scenario that enters the realm of 'beyond growth' options, EU GDP is $22 \%$ below BAU in 2050, showing essentially no growth over the period 2020-2050, but this is largely by construction as "employees seek to reduce hours worked in the formal economy ... in order to have more time for the family, engagement in society, volunteering and leisure ... [such that] hours worked per person are reduced by 20\%" (Distelkamp and Meyer, 2018, this issue). European $\mathrm{CO}_{2}$ emissions and primary material use fall dramatically in all scenarios, 
and in the rest of the world too in the Global Cooperation scenario. But even under Global Cooperation cumulative $\mathrm{CO}_{2}$ emissions exceed by 2040 the carbon budget that gives a $66 \%$ chance of staying below $2^{\circ} \mathrm{C}$ average warming.

In conclusion, it can be seen from this Special Issue that policy strategy at the EU level has recognised the desirability, as well as the challenges, of greatly increasing resource efficiency and moving towards a circular economy (CE), and have put in place numerous policies towards that end, which have in some cases be accompanied by voluntary actions and broader acceptance of resource efficiency measures in the business sector. However, the analysis has also revealed that: 1) policies to promote resource efficiency and $\mathrm{CE}$ are embedded in a complex system of competing policy domains and actor constellations, where resource efficiency policies may conflict with established structures; 2) the most ambitious resource efficiency policies and targets tend to be supported by soft, non-binding instruments, which may mean that adoption and implementation cannot be enforced and largely depend on alignment with national and regional policy agendas; 3 ) the negotiation process of policies may compromise the adoption and implementation of the most effective resource efficiency policies with the required stringency to bring about substantial changes; 4) forward thinking businesses may struggle under current economic conditions to make resource efficiency business models financially viable. The result is that the resource efficiency concepts that offer radical increases in resource efficiency are politically infeasible, while those that are feasible offer only incremental resource efficiency improvements, which are unlikely to bring about desired changes.

POLFREE, as summarised in the papers contained in the Special Issue, has identified policies and policy mixes that could achieve radical increases in resource efficiency of the kind envisaged by European policy makers, and the POLFREE scenarios have shown these to be economically advantageous overall, both with global cooperation toward such a goal, or Europe acting as a first-mover. In fact, the economic benefits are so pronounced that this is a significant incentive for European policy makers to take the political risks involved in turning the vision in their resource efficiency Roadmap into reality.

\section{REFERENCES}

Drummond, P. 2015 'Conclusions and Policy Insights', POLFREE Deliverable 4.6, https://www.ucl.ac.uk/polfree/publications/publications-2014/conclusions.pdf

EC (European Commission) 2011 'Roadmap to a Resource Efficient Europe', COM/2011/0571 final, http://ec.europa.eu/environment/resource_efficiency/about/roadmap/index_en.htm

Jäger, J. 2014, A Vision for a Resource-Efficient Economy, POLFREE Deliverable 2.2, https://www.ucl.ac.uk/polfree/publications/publications2014/POLFREE_Del._2.2_28.03KSC_FormatMay2014.pdf

Kammerlander, M. 2014 'Individual behavioural barriers to resource-efficiency', POLFREE Deliverable 1.6, https://www.ucl.ac.uk/polfree/publications/publications-2014/individualbehavioural-barriers-to-resource-efficiency

Kemp, R. and Dijk, M. 2013 'Analytical Framework of Drivers and Barriers to Resource Efficiency’, POLFREE Deliverable 1.1, 
https://www.ucl.ac.uk/polfree/publications/publications2014/Deliverable_1.1_Reformatted_May2014.pdf

Kemp, R., Dijk, M., Domenech, T., Wieser, H., Bahn-Walkowiak, B. and Weaver, P. 2014 'Synthesis report and conclusions about barriers and drivers', POLFREE Deliverable 1.7, https://www.ucl.ac.uk/polfree/publications/publications-2014/1.7

Wilts, H., Gries, N. von, Bahn-Walkowiak, B., O`Brien, M., Busemann, J., Domenech, T., Bleischwitz, R., and Dijk, M. 2015 'Policy Mixes for Resource Efficiency', POLFREE Deliverable D2.3, https://www.ucl.ac.uk/polfree/publications/publications-2014/policymixes-report 